\title{
Ocular effects of acute ingestion of Cola nitida (Linn) on healthy adult volunteers
}

\section{SA Igwea , DN Akunyilib and EU Ikonne}

a Department of Pharmacology and Therapeutics, College of Health Sciences, Ebonyi State University Teaching Hospital, Abakaliki, Nigeria

${ }^{\mathrm{b}}$ Department of Pharmacology and Therapeutics, College of Medicine, University of Nigeria Teaching Hospital, Enugu

${ }^{c}$ Department of Optometry, College of Medicine and Health Sciences, Abia State University, PMB 2000, Uturu, Nigeria

*Author for correspondence: <nwosuigwe@yahoo.com>

\begin{abstract}
Cola nitida Linn (Sterculiaceae) is an economic plant widely distributed throughout West Africa and possibly other parts of Africa. Members are malvalves because of their lobular nature. Because of its ubiquity, the plant plays an important role in commerce, and in social activities where it is casually eaten without prescription or restriction. The ethnopharmacological effects of bolus ingestion of $30 \mathrm{~g}$ of Cola nitida was investigated on visually acute and healthy volunteers in order to determine its ocular implications or effects. Results showed that Cola nitida had no effect on the pupil diameter, visual acuity and intraocular pressure but improved the near point of convergence by $43 \%$ and increased the amplitude of accommodation by $11 \%$ while existing heterophorias are ameliorated. The stimulating effect of Cola nitida might overcome asthenopic symptoms with convergence insufficiency and allows near work to be done without stress. Somnolence and ocular muscle imbalance common features of the elderly can be ameliorated or relieved.
\end{abstract}

Key words: acute ingestion Cola nitida imbalance, heterophorias, ocular muscle

\section{Introduction}

Cola nitida is an important economic crop growing in the forest areas of West Africa. It is closely related to cocoa, Theobroma cacao Linn, having about twenty-five species of the genus cola of which only five or six are edible seeds called nuts: Cola nitida, Cola acuminata, Cola verticillata, Cola anomala and Cola ballayi ${ }^{1}$.

In Nigeria, the Yoruba farmer in the Southwest recognizes at least four varieties of cola nuts, two of which are edible, namely Cola nitida and Cola acuminata which produce red, pink, brown and white nuts often in the same pod. The nuts of both species are easily differentiated on the basis of the number of cotyledons. For instances, Cola nitida nuts have only two cotyledons while Cola acuminata nuts have three or more cotyledons.

Cola nitida accounts for over $70 \%$ of the total cola production of West Africa, and has a higher export value than other species since the seeds contain a higher proportion of the stimulating constituents. The plant tastes fairly sweet and the fruits are longitudinally rogose and wrinkled, nodular in varying degree and dorsally keeled ${ }^{2}$. On the other hand, Cola acuminata produces white brown seeds which have three or more cotyledons in smooth pods. The 
fruit is comparatively smooth lacking dorsal keel and the seeds taste bitter ${ }^{3}$. The dry nuts can be ground into powder and used in the production of a whole variety of soft drinks.

Cola nut is generally supposed to contain about equal quantities of caffeine and theobromine which are responsible for its stimulating effects. ${ }^{3}$ Theophylline, caffeine and theobromine are three closely related alkaloids that occur in plants, widely distributed geographically and are known as methylated xanthines. These alkaloids share common pharmacological actions of therapeutic interest such as stimulating the central nervous system, (CNS), the kidney to produce diuresis, increasing the cardiac cycle and relaxing the bronchioles ${ }^{4}$.

Factors underlying the demand for cola nuts are numerous and include that the nuts are widely chewed as stimulants, as aids to digestion and for sustaining life in the absence of food. The nuts are also offered as valuable gifts among Igbos of Southeast Nigeria on social activities such as visits, marriages, naming ceremonies, meetings, funerals, et cetera. The nuts are also used as stabilizers in the dye industry, and as cosmetic or whitener for lips and teeth.

Since Cola nitida and indeed cola nuts are eaten casually and without prescription among the Nigerian population, no attempt has been made to document its effect on the ocular system and in our continued efforts to determine the enthnopharmacological effects of local food spices on ocular tissues, this study was undertaken using healthy volunteers, that is, individuals with good ocular health.

\section{Materials and Methods}

Cola nitida seeds were obtained from a local farmer in the university town of Uturu, Abia State, Southeast Nigeria. For purposes of consistency only one source of the Cola nitida was used. There was no prior extraction and the seeds were eaten whole because ordinarily the Igbos eat the seed raw for entertainment and leisure purposes. The seeds were weighed after removing the covers and then refrigerated until required for use.

Ten healthy volunteers of both sexes weighing between 52 and $62 \mathrm{~kg}$ (mean $60.2 \pm 1.5 \mathrm{~kg}$ ) aged between 30 and 45 years were screened and enlisted into the study from those who attended the optometry outreach organized by the Department of Optometry Clinic, Abia State University, Uturu. Each volunteer or participant was interviewed separately and information on sociodemographic data, and medical history was obtained. Informed verbal consent was elicited. Each participant was further subjected to screening consisting of ocular and visual examinations by the optometrists to ensure good ocular health, and confirm no refractive errors, or ocular pathologies which might introduce errors in the study. The nature of the study was further explained to the volunteers and those not willing to participate were excluded. Furthermore, any volunteer who was pregnant at the time of the study or were on any form of medication, oral or topical were further excluded from the study. Additionally, each subject or volunteer had a normal near point of convergence (NPC) of between 8 and $10 \mathrm{~cm}$ before the study.

Subjects had initial measurements done of the pupil size, near point of convergence (NPC), visual acuity (VA) at far and near, habitual phoria at far and near, the amplitude of accommodation (AA) and tonometry before commencement of protocol so as to establish their initial or baseline values, such that each volunteer would serve as his or her own control.

Cola nitida $30 \mathrm{~g}$ was then administered to each subject to be chewed as a bolus. Usually the Igbos either eat cola nut alone or with alligator pepper Afromomum melegueta or other pepperish pastries. For the purpose of the present study, Cola nitida was eaten alone. After ingestion, $50 \mathrm{ml}$ of potable water was given to each volunteer to flush the masticated substance down the gut. The subjects were allowed to rest for 90 minutes so that the effect of $C$. nitida could be felt in the ocular tissues and adnexa (preliminary experiments had suggested that the effects of the nuts were observable in ocular tissues 90 minutes after ingestion). Thereafter the above measurements were taken every ten minutes for the next two hours while the IOP was taken once daily at 10:00 am each day. 
Differences between preingestion values and those obtained after the ingestion of Cola nitida represented the effect of the substance of the particular visual function. Each reading was taken 5 times and the mean computed. Data were expressed as the mean \pm SEM. The results were further subjected to statistical analysis using ANOVA.

\section{Measurements}

a. The pupil size (pupil diameter) was measured in mm using the pupil distance rulers. Readings were taken at three different positions and the mean calculated.

b. The near point of convergence (NPC) was measured with the subjects fixating at the tip of a pencil positioned initially at $40 \mathrm{~cm}$, then adjusted towards the subject reported diplopia. The distance between the position of doubling and the central plane of the subject was measured with a meter rule in $\mathrm{cm}$ to give the NPC.

c. The visual acuity (VA) was measured for near and far respectively using the standard illuminated Snellen optotypes at appropriate measuring distances $(0.4 \mathrm{~m}$ for near and $6 \mathrm{~m}$ for far respectively).

d. The standard Von Graeffe phoria method was used.

e. The amplitude of accommodation (AA) was accomplished using the minus lens to the blur method. The amount of minus lens added to the blur points plus 2.50 to compensate for the reduced target gave the AA in diopters (D).

f. The intraocular pressure (IOP) was measured using the Schiotz tonometer with a weight of $5.5 \mathrm{~g}$, and $0.4 \%$ xylocaine solution. The value was read from the accompanying table and recorded in $\mathrm{mmHg}$. Measurements were taken at the same time each day (10:00 am local time) in order to avoid diurnal variation. The millimeter rule, was used for the measurement of the pupil diameter while the meter rule was used for the NPC. The Snellen distance chart and the reduced Snellen chart were used for the measurement of VA and the habitual pho-

\begin{tabular}{|c|c|c|}
\hline Preingestion & $\begin{array}{l}\text { 1st Phoria values } 90 \\
\text { min postingestion }\end{array}$ & $\begin{array}{l}\text { 2nd Phoria val- } \\
\text { ues } 30 \text { min after } \\
\text { (2hr) }\end{array}$ \\
\hline $0 \mathrm{~min}$ & $90 \mathrm{~min}$ & $120 \mathrm{~min}$ \\
\hline 2 exo & 1 exo & orho \\
\hline 4 exo & 2 exo & 3 exo \\
\hline 1 exo & ortho & ortho \\
\hline 2 exo & 1 exo & ortho \\
\hline 3 exo & 1 exo & ortho \\
\hline 2 exo & 3 exo & 2 eso \\
\hline 3 exo & 2 exo & ortho \\
\hline 3 exo & 2 exo & 2 exo \\
\hline 2 exo & 1 exo & ortho \\
\hline 1 exo & 1 exo & ortho \\
\hline \multicolumn{3}{|c|}{$\begin{array}{l}\text { ortho }=\text { orthophoria } \\
\text { eso }=\text { esophoria } \\
\text { exo }=\text { exophoria }\end{array}$} \\
\hline
\end{tabular}

\begin{tabular}{|c|c|c|}
\hline Preingestion & $\begin{array}{l}1^{\text {st }} \text { Phoria values } 90 \\
\text { min postingestion }\end{array}$ & $\begin{array}{l}2^{\text {nd }} \text { Phoria val- } \\
\text { ues } 30 \text { min after } \\
(2 \mathrm{hr})\end{array}$ \\
\hline $0 \mathrm{~min}$ & $90 \mathrm{~min}$ & $120 \mathrm{~min}$ \\
\hline 6 exo & 5 exo & 5 exo \\
\hline 1 exo & 1 exo & 1 eso \\
\hline 5 exo & 3 eso & 5 exo \\
\hline 4 exo & 2 exo & 5 exo \\
\hline 8 exo & 5 exo & 4 exo \\
\hline 7 exo & 5 exo & 6 exo \\
\hline 5 exo & 4 exo & 4 exo \\
\hline 2 exo & 3 eso & 2 eso \\
\hline 4 exo & 2 exo & 4 exo \\
\hline 5 exo & 4 exo & 5 ехо \\
\hline
\end{tabular}

ria. The Schiotz tomometer and $0.4 \%$ xylocaine solution were used in the determination of the intraocular pressure (IOP).

\section{Results}

Cola nitida Linn (Sterculiaceae), belongs to the phylum Angiospermae having a voucher number SP 371.K54 and classified as malvalves due to its lobular nature.

a. Pupil size: The mean initial pupil diameter was $3.5 \pm 0.1 \mathrm{~mm}$, and following bolus inges- 


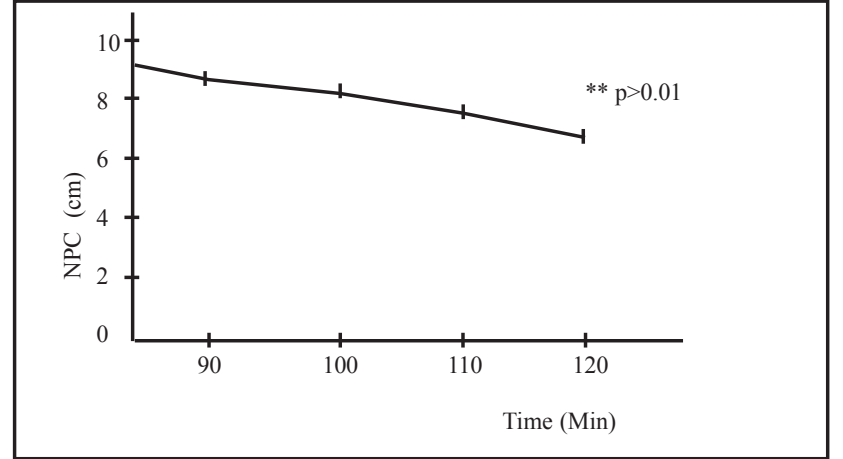

Figure 1: Effect of acute ingestion of Cola nitida (30g) on NPC of ten healthy volunteers. Each point represents the mean \pm SEM of 5 readings $(p>0.01)$. This improvement in NPC lasted for $2 \mathrm{hr}$ before returning to baseline values.

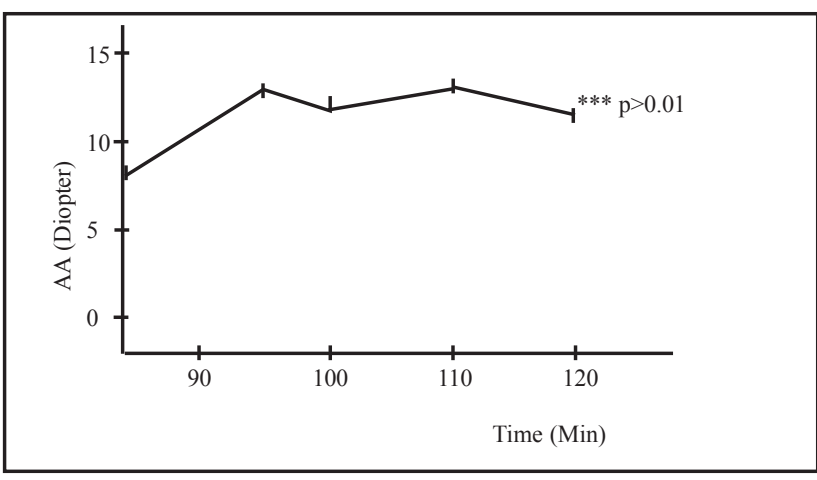

Figure 2: Changes in AA with time following acute ingestion of Cola nitida $(30 \mathrm{~g})$ by ten volunteers. Each point represents the mean \pm SEM of 5 readings $(p>0.01)$. The increase is small but persistent.

tion of Cola nitida, there was no change in the size of the pupil. This shows that Cola nitida has no short term effect on the pupil size.

b. $N P C$ : Each volunteer had a relatively normal NPC of between 8 and $10 \mathrm{~cm}$, and the mean NPC before the ingestion of Cola nitida was $9.6 \pm 0.5 \mathrm{~cm}$ and after the ingestion there was an improvement in the NPC and the mean was $5.5 \pm 0.3 \mathrm{~cm}$ or $43 \%$ advancement or improvement. Figure 1 shows that a significant improvement $(p>0.01)$ in NPC existed at the end of 120 minutes.

c. VA: All the subjects had normal VA at near (N5) and far (6/6) respectively before the commencement of protocol. These values did not change following bolus ingestion of Cola nitida.

d. AA: The volunteers had normal AA before the commencement of study and following acute ingestion of Cola nitida the mean AA increased from initial mean value of 7.34 \pm $0.02 \mathrm{D}$ to $8.25 \pm 0.5 \mathrm{D}$ or $11 \%$ increase. In
Figure 2, a significant but small and persistent increase $(p>0.01)$ in AA is presented at the peak effect.

e. Phoria status: Before the ingestion of Cola nitida $90 \%$ of the subjects were exophoric at far while the rest were esophoric at the vertical direction. However, following the ingestion of $30 \mathrm{~g}$ of Cola nitida the esophoric subjects remained so despite the changes in convergence while $80 \%$ of the exophoric subjects became orthophoric and the rest remaining exophoric. Furthermore, following the ingestion of Cola nitida all the exophoric subjects remained divergent (unchanged) while the esophoric subjects remained so (convergent) at the end of the study. The values are shown in Table 2 .

f. IOP: The mean IOP taken at 10:00 am local time was $22.62 \pm 1.31 \mathrm{mmHg}$ at the beginning of the study. This did not show any significant change taken at the same time at the end of the study, thus showing that Cola nitida has on average, no effect on the IOP.

\section{Discussion}

Cola nut (Cola nitida) is a surrogate of caffeine and the later is a known central nervous system stimulant ${ }^{3,5}$, and ingestion of cola nut is accompanied with signs of central nervous system stimulation such as insomnia or wakefulness.

In the present study which considered the effect of acute ingestion of Cola nitida on the visual system showed that the NPC improved by $43 \%$ while the AA increased by $11 \%$. These results are positively correlated $(r=0.92)$. On the other hand, pupil size, VA, near phoria and the intraocular pressure remained unaffected while the effect on distant (far) phoria tended towards correcting existing ocular muscle imbalance that is, orthoporia (Table 1).

The initial values of these visual functions served as an internal control for each volunteer. The use of placebo for the study would have introduced further complications.

Usually increase in accommodation would be associated with constriction of the pupil which is a miotic property and improvement in NPC would lead to convergence excess or exophoric and predispose the individual to headache and asthenopic symptoms during near 
work but because of the stimulating effect these symptoms are not manifested. Furthermore, the increased AA and the improved convergence would allow for near work without stress hence the use of cola nuts by the elderly Igbos during reading and writing for a long period without discomfort. Cola nut is for the elderly Igbo what coffee is to Europeans. Further work is indicated along this line.

Although a miotic effect was not manifested in the pupil size and, the VA remained unchanged despite the increased AA, hence there is no blurring of vision or impairment of vision or drowsiness when cola nut is ingested.

The effect on NPC possibly might be exploited in the management of convergence insufficiency which is a common phenomena among elderly patients. Somnolence also a common feature of the elderly, is inhibited when Cola nitida is ingested and thus would produce some degree of insomnia, wakefulness and alertness.

The resolving power of the eye (VA) was not in any way affected by the ingestion of Cola nitida $(30 \mathrm{~g})$ and as the pupil diameter of the subjects remained unchanged, it showed that Cola nitida had no effect on either the pupillary muscle sphincter or the dilator pupillae. Furthermore, as there was no effect on VA, consequently there were no changes in refractive status, hence no blurring of vision was recorded after ingesting Cola nitida, rather clear vision.

Finally, the NPC which plays an important role in the diagnosis of visual problems, and when it is lower than normal, as in the present study, the individual would experience convergence excess and poor depth perception. Additively, the increased AA plays a role in the convergence excess which may predispose to premature presbyopia which would make near work difficult, however, these symptoms are obstructed because of the stimulating effect of Cola nitida on the central nervous system.

We conclude that Cola nitida is an important and safe crop whose ethnopharmacological potentials can be exploited to improve the NPC when near work is being contemplated.

\section{References}

1. Ade A. Kola nut production and trade in Nigeria. Nigeria Institute of Social and Economic Research (NISER) Ibadan, 1982 pp 1 - 20 .

2. Akinyosoye VO. Senior Tropical Agriculture. Macmillan
Publishers Ltd. London 1984 pp 107 - 108.

3. Goldstein A, Kaizer S and Warren R. Psychotropoic effects of caffeine Pharmacol in man II, Alertness psychomotor coordination and mood. J Pharmacol Therapy 1967150146 - 151.

4. Beavo, JA Rogers NL, Crofford OB, Hardman JG, Sutherland EW and Newman EV. Effects of Xanthine derivatives on lipolysis and on adenesine 3,5 monophosphate, phosphodiesterase activity. Molecular Pharm 19706597 - 603.

5. Kordash TR, Van-Dellen RG and McCall JT. Theophylline concentrations in asthmatic patients after administering of aminophylline. J Am Med Assoc 1977238139 - 141. 